\title{
On a Question of Ameer Athavale
}

\author{
JIM GLEASON
}

\begin{abstract}
We answer the question posed by Athavale concerning the relationship of the Fredholm indices of a pure commuting subnormal tuple and its dual.
\end{abstract}

In this paper we shall answer the following question of Athavale, [2] in the affirmative.

Question. If $S$ is a subnormal m-tuple on $\mathcal{H}$ and $T$ its dual such that both $S$ and $T$ are Fredholm, is it true that

$$
\operatorname{ind}(S)=(-1)^{m+1} \operatorname{ind}(T) ?
$$

We begin by first establishing some notation. Let $S=\left(S_{1}, \ldots, S_{m}\right)$ be a pure subnormal tuple of operators on a Hilbert space $\mathcal{H}$ with minimal normal extension $N=\left(N_{1}, \ldots, N_{m}\right)$ acting on a Hilbert space $\mathcal{K} \supset \mathcal{H}$. Furthermore, let $T=\left(T_{1}, \ldots, T_{m}\right)$ be the dual of $S$ defined by $T_{j}=\left.N_{j}^{*}\right|_{\mathcal{K} \ominus \mathcal{H}}$ for all $j=1, \ldots, m$. In [3], Curto defines $S$ to be Fredholm if the associated Koszul complex,

$$
K(S, \mathcal{H}): 0 \rightarrow \Lambda^{0}(\mathcal{H}) \stackrel{\delta_{S, 0}}{\longrightarrow} \Lambda^{1}(\mathcal{H}) \stackrel{\delta_{S, 1}}{\longrightarrow} \cdots \stackrel{\delta_{S, d-1}}{\longrightarrow} \Lambda^{d}(\mathcal{H}) \rightarrow 0,
$$

is Fredholm and

$$
\operatorname{ind}(S):=\sum_{p=0}^{m}(-1)^{p} \operatorname{dim} H^{p}\left(\delta_{S}\right) .
$$

In order to answer the question, it is necessary to view the Fredholm tuple and its index in multiple ways. One such view of the index is to see it in terms of the Euler characteristic of the Koszul complex. By doing so, we can take advantage of the fields of homological algebras and K-theory which gives us the result from [1, Thm. I.1.7 
and Cor. I.1.8] that if

$$
0 \rightarrow\left(\mathfrak{X}^{\prime}, \delta^{\prime}\right) \rightarrow\left(\mathfrak{X}^{\prime \prime}, \delta^{\prime \prime}\right) \rightarrow(\mathfrak{X}, \delta) \rightarrow 0
$$

is a short exact sequence of Banach space complexes with $\left(\mathfrak{X}^{\prime}, \delta^{\prime}\right)$ being Fredholm, then $\left(\mathfrak{X}^{\prime \prime}, \delta^{\prime \prime}\right)$ is Fredholm if and only if $(\mathfrak{X}, \delta)$ is Fredholm. Furthermore, if all three are Fredholm then

$$
\operatorname{ind}\left(\mathfrak{X}^{\prime \prime}, \delta^{\prime \prime}\right)=\operatorname{ind}\left(\mathfrak{X}^{\prime}, \delta^{\prime}\right)+\operatorname{ind}(\mathfrak{X}, \delta) .
$$

So if $\mathcal{H}_{1}$ and $\mathcal{H}_{2}$ are two Hilbert spaces and $A$ is a tuple acting on $\mathcal{H}_{1} \oplus \mathcal{H}_{2}$ represented as $A=\left(\begin{array}{cc}A_{1} & A_{2} \\ 0 & A_{3}\end{array}\right)$ with $A_{1}$ being Fredholm, then $A$ is Fredholm if and only if $A_{3}$ is. In case both $A_{1}$ and $A_{3}$ are Fredholm,

$$
\operatorname{ind}(A)=\operatorname{ind}\left(A_{1}\right)+\operatorname{ind}\left(A_{3}\right) .
$$

In particular in the case where $S$ is a pure subnormal tuple with minimal normal extension $N$ and dual $T$ we have that $N=\left(\begin{array}{cc}S & A_{2} \\ 0 & T^{*}\end{array}\right)$ and thus

$$
\operatorname{ind}(N)=\operatorname{ind}(S)+\operatorname{ind}\left(T^{*}\right) .
$$

We must also study the Fredholm tuple by looking at the Dirac operator associated to the commuting tuple $S$ on a Hilbert space $\mathcal{H}$ defined by

$$
D_{S}:=\left.\left(\delta_{S}+\delta_{S}^{*}\right)\right|_{\Lambda^{\text {even }}(\mathcal{H})}: \Lambda^{\text {even }}(\mathcal{H}) \rightarrow \Lambda^{\text {odd }}(\mathcal{H}) .
$$

Since

$$
\begin{aligned}
H^{k}(\delta) & =\operatorname{ker}\left(\delta_{S, k}\right) / \operatorname{ran}\left(\delta_{S, k-1}\right) \cong \operatorname{ker}\left(\delta_{S, k}\right) \cap \operatorname{ran}\left(\delta_{S, k-1}\right)^{\perp} \\
& =\operatorname{ker}\left(\delta_{S, k}\right) \cap \operatorname{ker}\left(\left(\delta_{S, k-1}\right)^{*}\right)=\operatorname{ker}\left(\delta_{S, k}+\left(\delta_{S, k-1}\right)^{*}\right)
\end{aligned}
$$

we can see that

$$
\operatorname{dim}\left(\operatorname{ker}\left(D_{S}\right)\right)=\operatorname{dim}\left(\sum H^{\text {even }}\left(\delta_{S}\right)\right)
$$

and

$$
\operatorname{dim}\left(\operatorname{ker}\left(D_{S}^{*}\right)\right)=\operatorname{dim}\left(\sum H^{\text {odd }}\left(\delta_{S}\right)\right) .
$$

Thus $D_{S}$ is Fredholm if and only if $S$ is Fredholm and in the case that both are Fredholm we have that $\operatorname{ind}(S)=\operatorname{ind}\left(D_{S}\right)$. Therefore, since the Dirac operator of a normal tuple, $N$, is normal, we know that $\operatorname{ind}(N)=0$.

Combining these two views we see that $\operatorname{ind}(S)=-\operatorname{ind}\left(T^{*}\right)$ and from Proposition 9.1 in [3] we know that $\operatorname{ind}\left(T^{*}\right)=(-1)^{m} \operatorname{ind}(T)$ and we have the following theorem. 
Theorem. If $S$ is a subnormal m-tuple on $\mathcal{H}$ and $T$ its dual such that both $S$ and $T$ are Fredholm, then $\operatorname{ind}(S)=(-1)^{m+1} \operatorname{ind}(T)$.

\section{REFERENCES}

[1] C.-G. Ambrozie and F.-H. Vasilescu. Banach Space Complexes. Kluwer Academic Publishers, Dordrecht, 1995.

[2] A. Athavale. On the duals of subnormal tuples. Int. Eq. Operator Theory, 12 (1989), 305-323.

[3] R. E. Curto. Fredholm and inverible n-tuples of operators. The deformation problem. Trans. Amer. Math. Soc. 266 (1981).

\section{Jim Gleason,}

Department of Mathematics,

University of California,

Santa Barbara, CA 93106, USA

gleason@math.ucsb.edu

Received on 15 May 2001. 